\title{
Primary Prevention of Cancer-Related Lymphedema Using Preventive Lymphatic Surgery: Systematic Review and Meta-analysis
}

\author{
Pedro Ciudad ${ }^{1,2,3}$ Joseph M. Escandón ${ }^{4}$ Valeria P. \\ ${ }^{1}$ Department of Plastic, Reconstructive and Burn Surgery, Arzobispo \\ Loayza National Hospital, Lima, Peru \\ 2 Department of Plastic and Reconstructive Surgery, China Medical \\ University Hospital, Taichung, Taiwan \\ ${ }^{3}$ Academic Department of Surgery. School of Medicine Hipolito \\ Unanue. Federico Villarreal National University, Lima, Perú \\ ${ }^{4}$ Division of Plastic and Reconstructive Surgery, Department of \\ Surgery, Strong Memorial Hospital, University of Rochester Medical \\ Center, Rochester, New York, United States \\ Indian J Plast Surg 2022;55:18-25.
}

\author{
Address for correspondence Pedro Ciudad, MD, PhD, Department of \\ Plastic, Reconstructive and Burn Surgery, Arzobispo Loayza National \\ Hospital, Lima, Peru (e-mail: pciudad@hotmail.com). \\ ${ }^{5}$ Division of Plastic and Reconstructive Surgery, Department of \\ Surgery, Beth Israel Deaconess Medical Center, Harvard Medical \\ School, Boston, Massachusetts, United States \\ ${ }^{6}$ Department of Surgery "P.Valdoni," Unit of Plastic and \\ Reconstructive Surgery, Sapienza University of Rome, Policlinico \\ Umberto I, Rome, Italy
}

\author{
Abstract \\ Keywords \\ - "lymphedema" \\ (mesh) \\ - "lymphatic vessels" \\ (mesh) \\ - "primary prevention" \\ (mesh) \\ - "microsurgery" \\ (mesh) \\ - "lymph node \\ excision" (mesh)
}

Background Several studies have proven prophylactic lymphovenous anastomosis (LVA) performed after lymphadenectomy can potentially reduce the risk of cancerrelated lymphedema (CRL) without compromising the oncological treatment. We present a systematic review of the current evidence on the primary prevention of CRL using preventive lymphatic surgery (PLS).

Patients and Methods A comprehensive search across PubMed, Cochrane-EBMR, Web of Science, Ovid Medline (R) and in-process, SCOPUS, and ScienceDirect was performed through December 2020. A meta-analysis with a random-effect method was accomplished. Results Twenty-four studies including 1547 patients fulfilled the inclusion criteria. Overall, 830 prophylactic LVA procedures were performed after oncological treatment, of which 61 developed lymphedema.

The pooled cumulative rate of upper extremity lymphedema after axillary lymph node dissection (ALND) and PLS was 5.15\% (95\% Cl, 2.9\%-7.5\%; $p<0.01)$. The pooled cumulative rate of lower extremity lymphedema after oncological surgical treatment and PLS was $6.66 \%$ $(95 \% \mathrm{Cl}<1-13.4 \%$, $\mathrm{p}$-value $=0.5)$. Pooled analysis showed that PLS reduced the incidence of upper and lower limb lymphedema after lymph node dissection by 18.7 per 100 patients treated (risk difference [RD] $-18.7 \%, 95 \% \mathrm{Cl}-29.5 \%$ to $-7.9 \% ; p<0.001$ ) and by 30.3 per 100 patients treated (RD $-30.3 \%, 95 \% \mathrm{Cl}-46.5 \%$ to $-14 \% ; p<0.001$ ), respectively, versus no prophylactic lymphatic reconstruction.

Conclusions Low-quality studies and a high risk of bias halt the formulating of strong recommendations in favor of PLS, despite preliminary reports theoretically indicating that the inclusion of PLS may significantly decrease the incidence of CRL. published online

February 25, 2022
DOI https://doi.org/ $10.1055 / \mathrm{s}-0041-1740085$ ISSN $0970-0358$

\footnotetext{
(c) 2022. Association of Plastic Surgeons of India. All rights reserved. This is an open access article published by Thieme under the terms of the Creative Commons Attribution-NonDerivative-NonCommercial-License, permitting copying and reproduction so long as the original work is given appropriate credit. Contents may not be used for commercial purposes, or adapted, remixed, transformed or built upon. (https://creativecommons.org/ licenses/by-nc-nd/4.0/) Thieme Medical and Scientific Publishers Pvt. Ltd., A-12, 2nd Floor, Sector 2, Noida-201301 UP, India
} 


\section{Introduction}

Lymphedema occurs when there is lymphatic fluid accumulation and stasis. This triggers an inflammatory response in the interstitial compartment, ultimately causing adipose tissue proliferation and fibrous tissue deposition with mild-tosevere permanent edema of an affected body part and serious functional impairment. ${ }^{1-5}$ Additionally, the resulting edema can be further complicated by wound healing problems and infections, resulting in induration and disfigurement. ${ }^{1-5}$ Once lymphedema becomes clinically evident, its response to treatment is modest and tends to worsen, causing further destruction of the remaining functional lymphatic channels and irreversible fibrotic changes. ${ }^{1-3,5,6}$

With the inclusion of microsurgery, most efforts have been directed to the early secondary prevention of lymphedema or even tertiary prevention with excisional procedures in the setting of secondary lymphedema, ${ }^{7-11}$ which is more prevalent in industrialized countries following tumoral staging, lymph node dissection, and adjuvant radiotherapy for locoregional control of neoplastic disease. $^{12,13}$ Since the introduction of the concept of lymphatic microsurgical preventing healing approach (LYMPHA), ${ }^{14}$ the use of prophylactic lymphovenous anastomosis (LVA) after lymph node dissection (LND) has been implemented by several surgeons in recent reports in an attempt to reduce the risk of lymphedema without compromising the oncological treatment. ${ }^{15,16}$ However, there are few studies quantitatively synthesizing the magnitude of this intervention for both upper and lower extremity. Herein, we present a systematic review and meta-analysis of the current evidence on the primary prevention of cancer-related lymphedema (CRL) utilizing preventive lymphatic surgery (PLS).

\section{Patients and Methods}

\section{Literature Search}

A comprehensive systematic review and meta-analysis of the literature was performed based on the guidance of the Cochrane Handbook for Systematic Reviews of Interventions. ${ }^{17,18}$ The medical indices PubMed MEDLINE, Cochrane-EBMR, Web of Science, Ovid Medline(R), SCOPUS, and ScienceDirect were queried from database inception through December 2020. The following terms were searched in different combinations: ("Lymphedema" [Mesh]) OR ("Lymphedema/prevention and control" [Mesh]) OR ("Non-Filarial Lymphedema/prevention and control" [Mesh]) OR ("Non-Filarial Lymphedema" [Mesh]) AND ("Lymphedema/surgery" [Mesh]) OR (LVA) OR (Lymphovenous AND anastomosis) OR (Lymphaticovenous AND anastomosis) OR (lymph AND node AND transplant) OR (Lymph AND node AND transfer) AND (Prophylactic) OR (Prophylaxis) OR (Immediate) OR (Preventive) OR (Prevent) OR (Prevention). See - Supplementary Table S1 (online only), which displays the search strategy. A manual search of references was executed to find additional relevant studies.

\section{Selection Criteria}

Randomized and nonrandomized trials, observational cohort studies, case-control studies, and case series written in English were included. We included (i) single-arm studies reporting outcomes of microsurgical interventions directed toward the primary prevention of CRL, and (ii) double-arm studies encompassing a control group (no microsurgical intervention to prevent $\mathrm{CRL}$ ) and an experimental group receiving microsurgical interventions for the primary prevention of CRL. Primary prevention was defined as therapeutic interventions directed to prevent a disease from occurring; thus, the target population comprehended subjects with no clinical or paraclinical evidence of lymphedema. ${ }^{19,20}$ Studies solely addressing lymphatic mapping were excluded. Studies including interventions directed to secondary or tertiary lymphedema prevention, and single case reports, were also excluded.

\section{Data Extraction and Quality Assessment}

After duplicates were omitted, citations were screened based on title and abstract by two independent reviewers (J.M.E and V.P.B). Afterward, the reviewers performed a full-text assessment using the inclusion and exclusion criteria. Discrepancies were solved by the senior author (P.C.). Data extraction was performed by two independent reviewers. The number of patients, type of malignancies, LND, number of nodes removed, diagnostic modality, number of veins and lymphatic vessels anastomosed, type of LVAs, other reconstructive procedures, complications, and follow-up were extracted. The Oxford Centre for Evidence-Based Medicine (OCEBM) was used to provide critical appraisal of the level of evidence. ${ }^{21}$ The risk of bias within studies was assessed employing the Cochrane Collaboration tool. ${ }^{22,23}$

\section{Statistical Analysis}

Our primary outcome was to assess the rate of upper and lower extremity CRL after PLS, and to determine the risk difference (RD) of CRL following LND and PLS in comparison to no prophylactic lymphatic reconstruction. Therefore, for meta-analysis, an event was defined as a patient with a diagnosis of CRL. ${ }^{24}$ Jamovi 1.2.27.0 (Jamovi, Sydney, Australia) was used for statistical analysis. ${ }^{25-27}$ The pooled rate of postoperative CRL after PLS was estimated as the proportion of events over the total number of patients evaluated in the last follow-up. Due to the methodological heterogeneity within and between studies, a random-effect model meta-analysis of prevalence using the HartungKnapp-Sidik-Jonkman model was conducted on studies with a single intervention cohort. The effects size of studyspecific incidence was expressed by proportions $95 \%$ confidence intervals $(\mathrm{CI})$ and the overall pooled estimate with $95 \%$ binomial $\mathrm{Cl}^{28}$

In studies with both an experimental and a control group, a random-effect model meta-analysis for dichotomous outcomes using the Hartung-Knapp-Sidik-Jonkman method was accomplished to calculate the RD of developing lymphedema implementing PLS after LND. ${ }^{28-30}$ The RD was the calculated difference between the observed risks (proportions 
of individuals with the outcome of interest) in two cohorts (experimental vs. control). ${ }^{28-30}$

Heterogeneity was evaluated with the $Q$ statistic $p$ values and $I^{2}$ statistic. ${ }^{22}$ Substantial heterogeneity was considered if $I^{2}$ was greater than $50 \%{ }^{31}$ Statistical significance was considered at $p$-value $<0.05 .^{31}$ Publication bias was assessed qualitatively using a funnel plot graph and quantitatively with an Egger's regression test. ${ }^{32}$ We estimated the number of unpublished null studies (which fail to show a reduction in the incidence of CRL using PLS) required to eliminate the significance from our findings employing the Rosenthal's fail-safe $\mathrm{N}$ method.

A sensitivity analysis was conducted to assess the influence of certain features in the magnitude and precision of the overall prevalence of CRL following PLS. The following characteristics were excluded: $<10$ participants and preliminary reports.

\section{Results}

\section{Study Characteristic}

Our search yielded 678 citations, from which 159 duplicates were removed. Records were screened based on title and abstract, and 425 references were eliminated. Full-text assessment was performed on 94 collated reports, and 75 did not meet the inclusion criteria. A manual search of the references of included studies yielded five additional articles for review. Twenty-four studies fulfilled the inclusion criteria

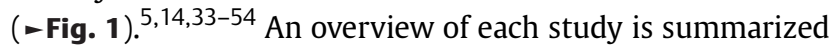
in - Supplementary Table S2 (available online only). Ten and six single-arm studies reporting outcomes of PLS for upper (-Supplementary Table 53; available online only) and lower (-Supplementary Table S4; available online only) extremity CRL were included, respectively. Seven and three double-arm studies reporting outcomes of PLS for upper ( - Supplementary Table 55; available online only) and lower extremity (-Supplementary Table S6; available online only) CRL were included, respectively.

Eighteen studies were observational studies, two were randomized control studies, one was a case series, and three were abstracts or conference presentations ( - Supplementary Table S2; available online only). The OCEBM level of evidence for the articles was as follows: eight articles had a level of evidence $3 \mathrm{~b}$ and sixteen a level of 4 .

\section{Patient Characteristics}

The qualitative analysis included 1547 patients (-Supplementary Table S2; available online only). The age of patients undergoing oncologic surgical treatment ranged from 18 to 87 years, and the follow-up period ranged from 6 to 156 months. Overall, 1247 patients (80.6\%) underwent axillary lymph node dissection (ALND), 1178 (75.6\%) for breast cancer and 69 (4.4\%) for melanoma. Three-hundred patients (19.4\%) underwent ilioinguinal, para-aortic, inguinofemoral lymph node dissection, and/or wide tissue excision of the inguinal region; the oncologic indications were highly heterogeneous (- Supplementary Table S2; available online only).
Overall, 830 prophylactic LVA procedures were performed after oncologic surgical treatment, of which 61 resulted in CRL during the follow-up period. Double-arm studies included 681 controls, of which 188 developed lymphedema during the follow-up period (-Supplementary Tables S3-S6; available online only).

Different lymphedema diagnostic modalities were reported. Five studies used a combination of methods while nineteen used a single diagnostic modality. Eight used volumetry, eight used circumferential measures, seven defined lymphedema clinically, four used lymphoscintigraphy, and four used bioimpedance spectroscopy (-Supplementary Table S2; available online only).

Six studies implemented the supermicrosurgery technique in which LVAs were performed in an end-to-end or end-to-side fashion when lymphatics and veins had a good size match. Twenty studies reported the use of sleeve LVAs when a substantial size mismatch occurred between the recipient vein and the available lymphatics. One study reported the use of S-LYMPHA technique (-Supplementary Tables S3-S6; available online only).

Complications were not ubiquitously reported. However, the most common complications were soft-tissue infections in 38 patients, lymphocele in seven patients, skin necrosis in five patients, venous backflow in one patient, transient arm swelling in two patients, and an infected seroma in one patient.

\section{Primary Prevention of CRL in Single Cohort Studies}

The pooled cumulative rate of upper extremity lymphedema after ALND and PLS was 5.15\% (95\% CI 2.9\%-7.5\%, $p<0.001)$ (-Supplementary Fig. S1; available online only). No significant heterogeneity was present across studies $\left(I^{2}=4.6 \%\right.$, $p=0.9$ ).

The pooled cumulative rate of lower extremity lymphedema after oncological surgical treatment (ilioinguinal, para-aortic, inguinofemoral lymph node dissection, or wide tissue excision) and PLS was 6.66\% (95\% CI $<1 \%-13.4 \%$, $p=0.05$ ) (-Supplementary Fig. S2; available online only). Considerable heterogeneity was present across studies $\left(I^{2}=61.11 \%, p=0.17\right)$.

When excluding studies with a sample size less than 10 and preliminary reports, the pooled rate of CRL was 5\% (95\% CI $2.5 \%-7.6 \%, p<0.001)$ and $6.9 \%(95 \% \mathrm{CI}<0.1 \%-14.8 \%$, $p=0.08)$ for upper and lower extremity, respectively.

\section{Primary Prevention of CRL in double-arm Studies}

The measure of effect used to compare the risk of developing CRL among double-arm studies was RD. The pooled analysis showed that PLS reduced the rate of upper limb lymphedema after ALND by 18.7 per 100 patients treated (RD - 18.7\%, 95\% CI - $29.5 \%$ to $-7.9 \% ; p<0.001 ; 10$ studies, grade $=$ low) (-Supplementary Fig. S3; available online only). Heterogeneity was substantial with significant clinical relevance $\left(I^{2}=70.7 \%, p=0.04\right)$. Pooled analysis showed that PLS reduced the rate of lower limb lymphedema after ilioinguinal lymph node dissection by 30.3 per 100 patients treated (RD $-30.3 \%$, $95 \% \mathrm{CI}-46.5 \%$ to $-14 \% ; p<.001 ; 3$ studies, grade $=$ low $)$ 


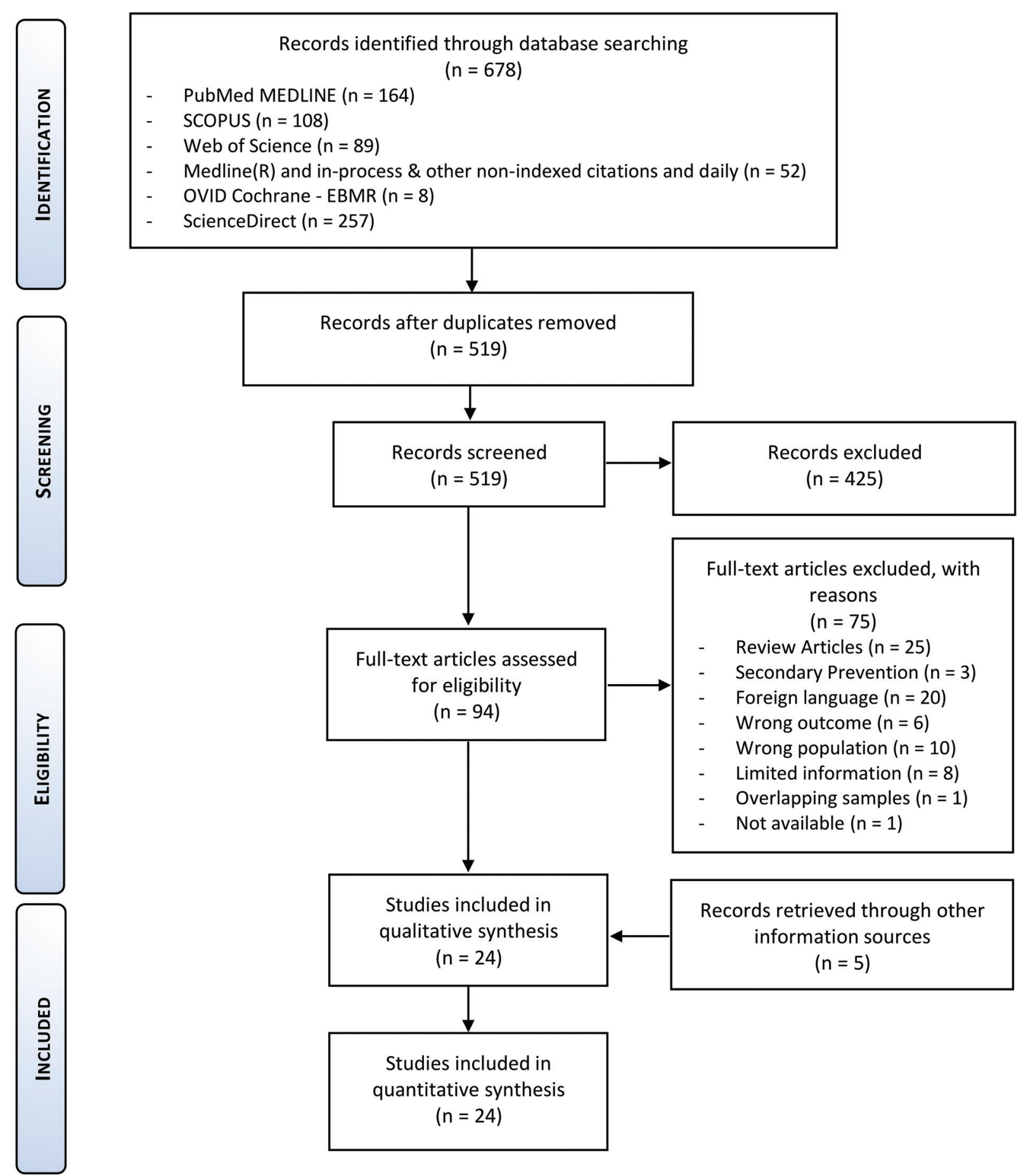

Fig. 1 Preferred Reporting Items for Systematic Reviews and Meta-Analyses (PRISMA) flow diagram.

(-Supplementary Fig. S4; available online only). No significant heterogeneity was present across studies $\left(I^{2}=37.32 \%\right.$, $p=0.29$ )

\section{Publications Bias}

The funnel plot of the different meta-analyses showed asymmetry (-Supplementary Fig. S5; available online only). Quantitatively, meta-analysis for single-arm studies using PLS for the primary prevention of upper extremity (Egger's test $p$-value 0.519 ) and lower extremity lymphedema (Egger's test $p$-value 0.184 ) did not display significant bias. Similarly, the meta-analyses of dichotomous outcomes for double-arm studies of upper extremity (Egger's test $p$ value 0.445 ) and lower extremity lymphedema (Egger's test $p$-value 0.831 ) using PLS did not exhibit significant bias. We estimated that 119 and 22 unpublished null studies would be required to disregard the significance of the conclusions from the meta-analyses of dichotomous outcomes for upper and lower extremity, respectively.

Included articles did not report the method used to generate the allocation sequence, conceal the allocation sequence, or blind study participants and personnel. Due 


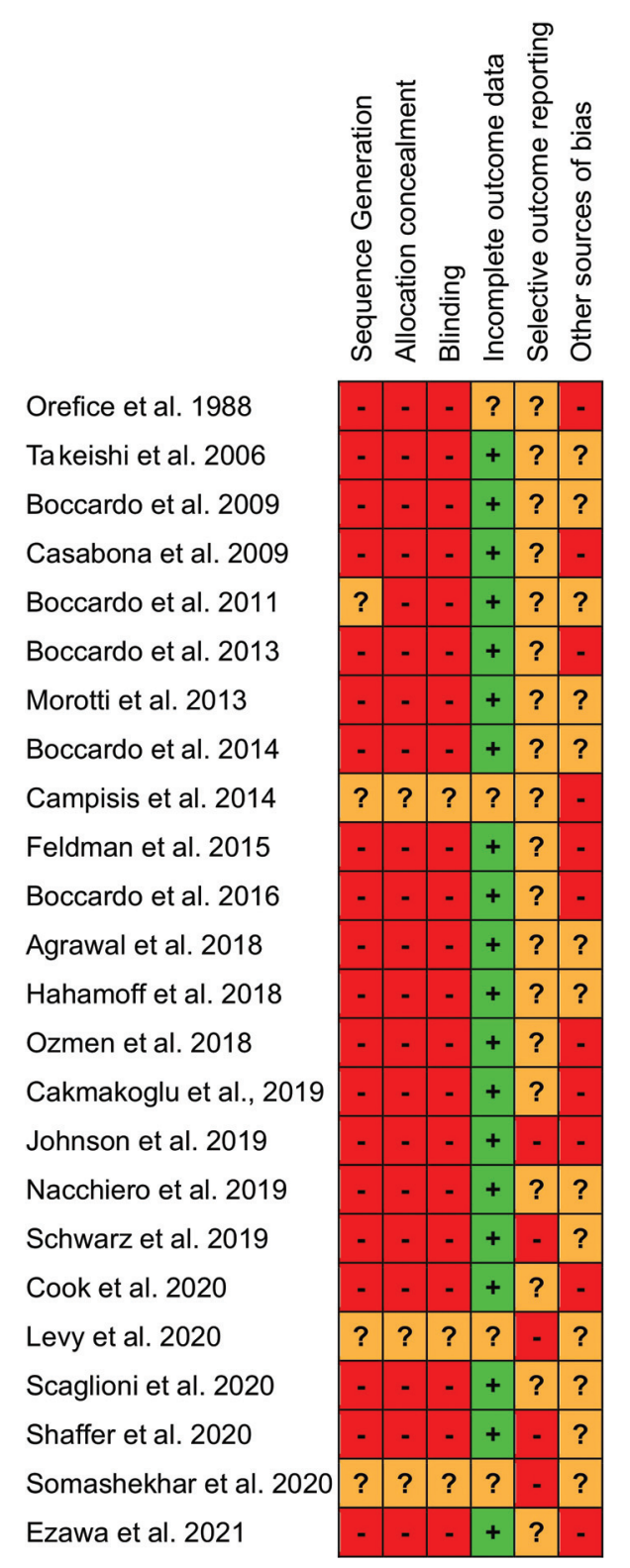

Fig. 2 Risk of bias analysis of included studies. (Red [-]: high risk of bias; yellow (?): unclear risk of bias; green $(+)$ : low risk of bias).

to its investigational nature, the experimental groups in some studies had a lower sample size in comparison to control groups; however, the completeness of outcome data did not seem to be compromised. All articles were highly biased, and the protocols of the included studies were not documented on international registries (-Fig. 2).

\section{Discussion}

Ninety-nine percent of secondary nonfilarial lymphedema results from the treatment of malignancies. ${ }^{55,56}$ Therefore, strategies directed to treat CRL beyond the secondary or tertiary prevention are currently considered indispensable. ${ }^{44}$ Since Boccardo et al published their study LYMPHA, in which breast CRL (BCRL) was prevented in a cohort of 18 patients who underwent prophylactic LVAs after ALND, ${ }^{49}$ further studies have been published portraying promising results using preventive LVA to preclude secondary lymphedema. ${ }^{5,51,53,54}$

A recent meta-analysis reported that the pooled incidence of lymphedema after ALND was $14.1 \%$, and $33.4 \%$ when ALND was associated with regional lymph node radiation (RLNR). ${ }^{57}$ In our meta-analysis, $22.39 \%$ of patients in the control group developed lymphedema after ALND, which lies within previously reported data, suggesting an acceptable external validity to overall patient population despite the undetermined risk of selecting outcome reporting. In fact, the incidence of BCRL 5 years postoperatively has been documented to be from $3 \%$ to $42.2 \%$ depending on several factors such as the diagnostic methods and the adjuvant oncologic treatment. $^{58}$

With this precedent, several techniques like axillary reverse mapping (ARM) have demonstrated to improve the primary prevention of BCRL. ${ }^{44,59}$ For instance, the incidence of upper extremity lymphedema was approximately 1 to $2 \%$ when patients underwent sentinel lymph node biopsy (SLNB) in association with ARM and 2 to $6 \%$ when ALND was performed in conjunction with ARM. ${ }^{44,59-61}$ Therefore, further reduction of the risk of lymphedema may be achieved with the implementation of prophylactic LVAs, as we found in the present meta-analysis that PLS significantly reduced the risk of CRL after ALND in comparison to no intervention ( $\mathrm{RD}-30.3 \%, 95 \% \mathrm{CI}-46.5 \%$ to $-14 \%$ ).

Since first introduced by Orefice and colleagues in $1988,{ }^{43}$ lower extremity PLS has become more popular. Hyngstrom et al reported that after superficial inguinofemoral LND for the locoregional control of melanoma, lymphedema at a 12month follow up period occurred in $61.5 \%$ of patients. ${ }^{62}$ Similarly, in a recent meta-analysis, the pooled incidence of lower limb lymphedema in women who specifically underwent inguinofemoral lymph node dissection for the treatment of vulva cancer was estimated at $32.1 \%(95 \% \mathrm{CI}$ 20.2-44.0). ${ }^{63}$ Remarkably, our findings indicated that PLS reduced the risk of lymphedema after ilioinguinal LND in comparison to no prophylactic intervention (risk difference $30.3 \%, 95 \% \mathrm{Cl}-46.5 \%$ to $-14 \%$ ), with a pooled cumulative incidence for lower extremity lymphedema of $6.66 \%$ (95\% $\mathrm{CI}<1 \%-13.4 \%$ ).

Despite overcoming the limitation previously reported in different reviews by segregating the outcomes for upper and lower limb CRL, ${ }^{16}$ several other limitations were found in our meta-analysis. For instance, the different levels and regions of LND, types of LVA (E-E or telescoping), past radiation therapy, diagnostic modalities, and the different follow-up periods provided a very heterogenous sample, affecting the overall methodology and increasing the risk of bias toward better outcomes, especially in cases where selective outcome reporting was uncertain. Remarkably, the diagnostic assessment of lymphedema was severely heterogeneous with less reliable tools (e.g., volumetry, clinical examination, and circumferential measures) being implemented in the majority of studies, which increased the risk of bias in the setting of unblinded protocols. Indeed, only $16.6 \%$ of the studies used reliable methods with an appropriate sensitivity and specificity like lymphoscintigraphy. ${ }^{64,65}$ 
Overall, only $33.3 \%$ of included studies reported outcomes with an average follow-up of 2 years or more. This may affect in great part the prevalence of lymphedema, as a minimum follow-up of 3 years after starting oncologic treatment should be contemplated to satisfactorily identify patients suffering with CRL in subclinical and early stages as well as any aftermath related to the recurrence of neoplastic diseases. $^{56,66,67}$

Complications associated with PLS in the form of prophylactic LVA are in the majority Clavien-Dindo classification I and II. ${ }^{68,69}$ This includes surgical site infection, lymphocele, skin necrosis, and transient swelling, which may be attributed in great part to the concurrent oncologic excision and lymphadenectomy performed in the same surgical time of the LVA. PLS by means of LVA may add 15 to 45 minutes to the surgical time if performed orthotopically or 46 to 120 minute if performed heterotopically. ${ }^{16}$ Nonetheless, further studies are required to unveil the effects of additional operative time for LVA and the morbidity associated with the overall procedure.

In a recent economic analysis of PLS among breast cancer patients receiving mastectomy with ALND, an estimated $\$ 7,646.65$ cost saving per patient was calculated in patients undergoing prophylactic LVA. This sum represented $42 \%$ of the total cost per patient in whom no prophylactic intervention was performed. ${ }^{70}$ Likewise, in a cost-utility analysis presented by Johnson et al, immediate lymphatic reconstruction after ALND was more cost-effective in comparison to ALND alone, with an incremental cost-utility ratio of $\$ 1587.73$ per quality-adjusted life year. ${ }^{71}$ Similarly, in patients undergoing RLNR, the incorporation of immediate lymphatic reconstruction provided an incremental cost-utility ratio of $\$ 699.84$ per quality-adjusted life year. ${ }^{71}$ No reported cost-effective analysis was found in literature regarding PLS on the lower extremity; however, from a cost minimization perspective, the inclusion of LVA for the prevention of CRL seems promising. In this setting, although PLS is not a therapeutic approach that can be graded with a strong recommendation given the low confidence in the effect estimates. ${ }^{72}$ PLS has shown to significantly decrease the incidence of lymphedema and decrease the economic burden of this disease with a low associated morbidity despite the high risk of bias of published reports. ${ }^{73}$

To our knowledge, there are no published cases reporting the transfer of vascularized lymph node flaps, used solely for the primary prevention of CRL. This, due to the risk for complications and the invasive nature of these procedures, make them less appealing from a preventive standpoint. In this review, Scaglioni et al presented a series of seven patients undergoing margin-free surgical resection for sarcomatous tumors along the groin and/or adductors compartment. Following resection, patients underwent reconstruction with a superficial circumflex iliac artery perforator (SCIP) flap with lymphatic preservation and at least one LVA at the level of the knee. Lymphoscintigraphy was performed at 6 months postoperatively showing no signs of lymphedema. ${ }^{47}$

Similarly, Gentileschi et al reported a cohort of patients undergoing bilateral groin dissection in which a pedicled SCIP flap with lymphatic preservation was used in one side, while the contralateral side was closed customarily. ${ }^{74}$ Postoperatively, the limbs treated with the SCIP flap showed no pathological swelling while the control limbs displayed a significantly increased postoperative volume and moderateto-severe signs of lymphedema. Certainly, flaps with lymphatic preservation seem to be a feasible alternative for the primary prevention of CRL. However, the surgeon must accomplish a thorough patient selection process and a benefit-risk analysis if a chimeric flap with lymphatic preservation or lymph node transfer is intended to prevent lymphedema given the associated morbidity of these procedures.

\section{Limitations}

The included studies were mostly of low quality which caused a significant heterogeneity in type of cancer, lymphadenectomy technique, location of LND, rate of adjuvant radiotherapy, and lymphedema diagnostic modality. Given that PLS is presently attaining responsiveness, there is a potential risk of publication bias, as studies may be discontinued or not published if unsuccessful results ensue. ${ }^{75}$ A prospective protocol was established and accepted by all the reviewers; however, it was not registered.

\section{Conclusions}

Given the safety profile, effectiveness, and feasibility of LVA, preliminary reports theoretically favor the inclusion of PLS for primary prevention of CRL. Nonetheless, low-quality studies with great heterogeneity, high risk of bias within studies, short follow-up periods, and variability among diagnostic modalities hinder the formulation of strong recommendations in favor of this intervention. High-quality studies are necessary to determine evidence-based recommendations regarding the use of preventive lymphatic surgery.

\section{Ethical Statement}

The authors are accountable for all aspects of the work in ensuring that questions related to the accuracy or integrity of any part of the work are appropriately investigated and resolved.

\section{Funding}

None of the authors received any funds or has any financial interests to disclose for the research, authorship, and publication of this article.

\section{Conflict of Interest}

None declared.

\section{References}

1 Ciudad P, Sabbagh MD, Agko M, et al. Surgical management of lower extremity lymphedema: a comprehensive review. Indian J Plast Surg 2019;52(01):81-92

2 Ghanta S, Cuzzone DA, Torrisi JS, et al. Regulation of inflammation and fibrosis by macrophages in lymphedema. Am J Physiol Heart Circ Physiol 2015;308(09):H1065-H1077 
3 Kung TA, Champaneria MC, Maki JH, Neligan PC. Current concepts in the surgical management of lymphedema. Plast Reconstr Surg 2017;139(04):1003e-1013e

4 Benoit L, Boichot C, Cheynel N, et al. Preventing lymphedema and morbidity with an omentum flap after ilioinguinal lymph node dissection. Ann Surg Oncol 2005;12(10):793-799

5 Boccardo F, Valenzano M, Costantini S, et al. LYMPHA technique to prevent secondary lower limb lymphedema. Ann Surg Oncol 2016;23(11):3558-3563

6 Leung EYL, Tirlapur SA, Meads C. The management of secondary lower limb lymphoedema in cancer patients: a systematic review. Palliat Med 2015;29(02):112-119

7 Gilbert A, O'Brien BMC, Vorrath JW, Sykes PJ. Lymphaticovenous anastomosis by microvascular technique. Br J Plast Surg 1976;29 (04):355-360

8 Forte AJ, Khan N, Huayllani MT, et al. Lymphaticovenous anastomosis for lower extremity lymphedema: a systematic review. Indian J Plast Surg 2020;53(01):17-24

9 Laine JB, Howard JM. Experimental lymphatico-venous anastomosis. Surg Forum 1963;14(14):111-112

10 Ciudad P, Agko M, Huang TCT, et al. Comprehensive multimodal surgical treatment of end-stage lower extremity lymphedema with toe management: The combined Charles,' Homan's, and vascularized lymph node transfer (CHAHOVA) procedures. J Surg Oncol 2019;119(04):430-438

11 Ciudad P, Manrique OJ, Bustos SS, et al. Single-stage VASERassisted liposuction and lymphatico-venous anastomoses for the treatment of extremity lymphedema: a case series and systematic review of the literature. Gland Surg 2020;9(02): 545-557

12 Laredo J, Lee BB. Lymphedema. In: Mowatt-Larssen E, Desai SS, Dua A, Shortell CE, eds. Phlebology, Vein Surgery and Ultrasonography: Diagnosis and Management of Venous Disease. 1st ed. Switzerland: Springer International Publishing; 2014:327-340

13 Manrique OJ, Bustos SS, Ciudad P, et al. Overview of lymphedema for physicians and other clinicians: a review of fundamental concepts. Mayo Clin Proc 2020;x:x

14 Boccardo F, Casabona F, De Cian F, et al. Lymphatic microsurgical preventing healing approach (LYMPHA) for primary surgical prevention of breast cancer-related lymphedema: over 4 years follow-up. Microsurgery 2014;34(06):421-424

15 Campisi CC, Ryan M, Boccardo F, Campisi C. LyMPHA and the prevention of lymphatic injuries: a rationale for early microsurgical intervention. J Reconstr Microsurg 2014;30(01):71-72

16 Jørgensen MG, Toyserkani NM, Sørensen JA. The effect of prophylactic lymphovenous anastomosis and shunts for preventing cancer-related lymphedema: a systematic review and meta-analysis. Microsurgery 2018;38(05):576-585

17 Shamseer L, Moher D, Clarke M, et al; PRISMA-P Group. Preferred reporting items for systematic review and meta-analysis protocols (PRISMA-P) 2015: elaboration and explanation. BMJ 2015; 350:g7647

18 Moher D, Shamseer L, Clarke M, et al; PRISMA-P Group. Preferred reporting items for systematic review and meta-analysis protocols (PRISMA-P) 2015 statement. Syst Rev 2015;4(01):1

19 Simeonsson RJ. Primary, secondary, and tertiary prevention in early intervention. J Early Interv 1991;15(02):124-134

20 Pigeot I, De Henauw S, Foraita R, Jahn I, Ahrens W. Primary prevention from the epidemiology perspective: three examples from the practice. BMC Med Res Methodol 2010;10:10

21 Durieux N, Vandenput S, Pasleau F. Médecine factuelle: la hiérarchisation des preuves par le Centre for Evidence-Based Medicine d'oOford [OCEBM levels of evidence system]. Rev Med Liege 2013;68(12):644-649

22 Higgins JPT, Thomas J, Chandler J, Cumpston M, Li T, Page MJ, Welch VA, (editors). Cochrane Handbook for Systematic Reviews of Interventions. 2nd Edition Chichester (UK): John Wiley \& Sons; 2019
23 Higgins JPT, Altman DG, Gøtzsche PC, et al; Cochrane Bias Methods Group Cochrane Statistical Methods Group. The Cochrane Collaboration's tool for assessing risk of bias in randomised trials. BMJ 2011;343(7829):d5928

24 Borges Migliavaca C, Stein C, Colpani V, Barker TH, Munn Z, Falavigna MPrevalence Estimates Reviews - Systematic Review Methodology Group (PERSyst) How are systematic reviews of prevalence conducted? A methodological study. BMC Med Res Methodol 2020;20(01):96

25 R Core Development Team. R: A Language and environment for statistical computing. (Version 4.0) [Computer Software]. Accessed September 14, 2021 at: https://cran.r-project.org

26 The Jamovi Project. Jamovi (Version 1.6) [Computer Software] Accessed September 14, 2021 at: https://www.jamovi.org

27 Viechtubauer W. Conducting Meta-Analysis in R with the metafor package. J Stat Softw 2010;36:1-48

28 IntHout J, Ioannidis JP, Borm GF. The Hartung-Knapp-Sidik-Jonkman method for random effects meta-analysis is straightforward and considerably outperforms the standard DerSimonian-Laird method. BMC Med Res Methodol 2014;14:25

29 Riley RD, Higgins JPT, Deeks JJ. Interpretation of random effects meta-analyses. BMJ 2011;342:d549

30 Bonett DG, Price RM. Meta-analysis methods for risk differences. Br J Math Stat Psychol 2014;67(03):371-387

31 Deeks J, Higgins J, Altman D. Chapter 10: Analysing data and undertaking meta-analyses. In: Higgins JPT, Chandler J, Cumpston M, Li T, Page MJ, Welch VA, (editors). Cochrane Handbook for Systematic Reviews of Interventions. 2nd Edition Chichester (UK): John Wiley \& Sons; 2019:241-280

32 Egger M, Davey Smith G, Schneider M, Minder C. Bias in metaanalysis detected by a simple, graphical test. BMJ 1997;315 (7109):629-634

33 Feldman S, Bansil H, Ascherman J, et al. Single institution experience with lymphatic microsurgical preventive healing approach (LYMPHA) for the primary prevention of lymphedema. Ann Surg Oncol 2015;22(10):3296-3301

34 Agrawal J, Mehta S, Goel A, Pande PK, Kumar K. Lymphatic microsurgical preventing healing approach (LYMPHA) for prevention of breast cancer-related lymphedema-A preliminary report. Indian J Surg Oncol 2018;9(03):369-373

35 Hahamoff M, Gupta N, Munoz D, et al. A lymphedema surveillance program for breast cancer patients reveals the promise of surgical prevention. J Surg Res 2019;244:604-611

36 Ozmen T, Lazaro M, Zhou Y, Vinyard A, Avisar E. Evaluation of simplified lymphatic microsurgical preventing healing approach (S-Lympha) for the prevention of breast cancer-related clinical lymphedema after axillary lymph node dissection. Ann Surg 2019;270(06):1156-1160

37 Cakmakoglu C, Kwiecien GJ, Schwarz GS, Gastman B. Lymphaticovenous bypass for immediate lymphatic reconstruction in locoregional advanced melanoma patients. J Reconstr Microsurg 2020;36(04):247-252

38 Ezawa M, Sasaki H, Yamada K, et al. Long term outcomes from lymphatic venous anastomosis after total hysterectomy to prevent postoperative lymphedema in lower limb. BMC Surg 2019;19 (01):177

39 Nacchiero E, Maruccia M, Vestita M, Elia R, Marannino P, Giudice G. Multiple lymphatic-venous anastomoses in reducing the risk of lymphedema in melanoma patients undergoing complete lymph node dissection. A retrospective case-control study. J Plast Reconstr Aesthet Surg 2019;72(04):642-648

40 Johnson AR, Fleishman A, Tran BNN, et al. Developing a lymphatic surgery program: a first-year review. Plast Reconstr Surg 2019; 144(06):975e-985e

41 Schwarz GS, Grobmyer SR, Djohan RS, et al. Axillary reverse mapping and lymphaticovenous bypass: Lymphedema prevention through enhanced lymphatic visualization and restoration of flow. J Surg Oncol 2019;120(02):160-167 
42 Cook JA, Sasor SE, Loewenstein SN, et al. Immediate lymphatic reconstruction after axillary lymphadenectomy: a single-institution early experience. Ann Surg Oncol 2021;28(03):1381-1387

43 Orefice S, Conti AR, Grassi M, Salvadori B. The use of lymphovenous anastomoses to prevent complications from ilio-inguinal dissection. Tumori 1988;74(03):347-351

44 Shaffer K, Cakmakoglu C, Schwarz GS, et al. Lymphedema prevention surgery: improved operating efficiency over time. Ann Surg Oncol 2020;27(12):4695-4701

45 Somashekhar S, Ashok B, Rohit Kumar C, Zaveri S, Ashwin K. Lymphatic microsurgical preventive healing approach (LYMPHA) - Lending a helping hand for prevention of enlarging arm. Ann Surg Oncol 2020;27(12):231-671

46 Levy AS, Peysakhovich A, Taback B, Ascherman JA, Rohde CH. Lymphatic microsurgical preventive healing approach (LYMPHA) for the primary prevention of lymphedema: a 3-year follow-up matched cohort study. Am Assoc Hand Surg 2020;22(06):3296

47 Scaglioni MF, Meroni M, Fritsche E, Fuchs B. Combined pedicled superficial circumflex iliac artery perforator (SCIP) flap with lymphatic tissue preservation and lymphovenous anastomosis (LVA) for defect reconstruction and lymphedema-lymphocele prevention in thigh sarcoma surgery: Preliminary results. J Surg Oncol 2021;123(01):96-103

48 Takeishi M, Kojima M, Mori K, Kurihara K, Sasaki H. Primary intrapelvic lymphaticovenular anastomosis following lymph node dissection. Ann Plast Surg 2006;57(03):300-304

49 Boccardo F, Casabona F, De Cian F, et al. Lymphedema microsurgical preventive healing approach: a new technique for primary prevention of arm lymphedema after mastectomy. Ann Surg Oncol 2009;16(03):703-708

50 Casabona F, Bogliolo S, Valenzano Menada M, Sala P, Villa G, Ferrero S. Feasibility of axillary reverse mapping during sentinel lymph node biopsy in breast cancer patients. Ann Surg Oncol 2009;16(09):2459-2463

51 Boccardo FM, Casabona F, Friedman D, et al. Surgical prevention of arm lymphedema after breast cancer treatment. Ann Surg Oncol 2011;18(09):2500-2505

52 Boccardo F, De Cian F, Campisi CC, et al. Surgical prevention and treatment of lymphedema after lymph node dissection in patients with cutaneous melanoma. Lymphology 2013;46(01):20-26

53 Morotti M, Menada MV, Boccardo F, et al. Lymphedema microsurgical preventive healing approach for primary prevention of lower limb lymphedema after inguinofemoral lymphadenectomy for vulvar cancer. Int J Gynecol Cancer 2013;23(04):769-774

54 Campisi CC, Campisi CA, Boccardo F, Ryan M. Microsurgery for prevention and early treatment of lymphatic disorders after nodal dissection. J Am Coll Surg 2014;219(Suppl 4):E198

55 Cormier JN, Askew RL, Mungovan KS, Xing Y, Ross MI, Armer JM. Lymphedema beyond breast cancer: a systematic review and meta-analysis of cancer-related secondary lymphedema. Cancer 2010;116(22):5138-5149

56 Rodriguez JR, Fuse Y, Yamamoto T. Microsurgical strategies for prophylaxis of cancer-related extremity lymphedema: a comprehensive review of the literature. J Reconstr Microsurg 2020;36 (07):471-479

57 Johnson AR, Kimball S, Epstein S, et al. Lymphedema incidence after axillary lymph node dissection: quantifying the impact of radiation and the lymphatic microsurgical preventive healing approach. Ann Plast Surg 2019;82(4S, Suppl 3)S234-S241

58 Ribeiro Pereira ACP, Koifman RJ, Bergmann A. Incidence and risk factors of lymphedema after breast cancer treatment: 10 years of follow-up. Breast 2017;36:67-73
59 Ochoa D, Korourian S, Boneti C, Adkins L, Badgwell B, Klimberg VS. Axillary reverse mapping: five-year experience. Surgery 2014; 156(05):1261-1268

60 Tummel E, Ochoa D, Korourian S, et al. Does axillary reverse mapping prevent lymphedema after lymphadenectomy? Ann Surg 2017;265(05):987-992

61 Yue T, Zhuang D, Zhou P, et al. A prospective study to assess the feasibility of axillary reverse mapping and evaluate its effect on preventing lymphedema in breast cancer patients. Clin Breast Cancer 2015;15(04):301-306

62 Hyngstrom JR, Chiang YJ, Cromwell KD, et al. Prospective assessment of lymphedema incidence and lymphedema-associated symptoms following lymph node surgery for melanoma. Melanoma Res 2013;23(04):290-297

63 Huang J, Yu N, Wang X, Long X. Incidence of lower limb lymphedema after vulvar cancer: A systematic review and meta-analysis. Medicine (Baltimore) 2017;96(46):e8722

64 Liang Z-Y, Long X, Yu N-Z, Huang J-Z. Diagnostic workup of lymphedema. Plast Aesthetic Res 2019;6(23):3-9

65 Forte AJ, Boczar D, Huayllani MT, Lu X, Ciudad P. Lymphoscintigraphy for evaluation of lymphedema treatment: a systematic review. Cureus 2019;11(12):e6363

66 PetrekJA, Senie RT, Peters M, Rosen PP. Lymphedema in a cohort of breast carcinoma survivors 20 years after diagnosis. Cancer 2001; 92(06):1368-1377

67 Johansson K, Branje E. Arm lymphoedema in a cohort of breast cancer survivors 10 years after diagnosis. Acta Oncol 2010;49 (02):166-173

68 Rosian K, Stanak M. Efficacy and safety assessment of lymphovenous anastomosis in patients with primary and secondary lymphoedema: A systematic review of prospective evidence. Microsurgery 2019;39(08):763-772

69 Clavien PA, Barkun J, de Oliveira ML, et al. The Clavien-Dindo classification of surgical complications: five-year experience. Ann Surg 2009;250(02):187-196

70 Squitieri L, Rasmussen PW, Patel KM. An economic analysis of prophylactic lymphovenous anastomosis among breast cancer patients receiving mastectomy with axillary lymph node dissection. J Surg Oncol 2020;121(08):1175-1178

71 Johnson AR, Asban A, Granoff MD, et al. Is immediate lymphatic reconstruction cost-effective? Ann Surg 2019 (e-pub ahead of print). Doi: 10.1097/sla.0000000000003746

72 Alexander PE, Brito JP, Neumann I, et al. World Health Organization strong recommendations based on low-quality evidence (study quality) are frequent and often inconsistent with GRADE guidance. J Clin Epidemiol 2016;72:98-106

73 Agency for Healthcare Research and Quality (US) Appendix A: How the U.S. Preventive Services Task Force Grades Its Recommendations. In: The Guide to Clinical Preventive Services 2012: Recommendations of the U.S. Preventive Services Task Force. Accessed September 14, 2021 at: https://www.ncbi.nlm.nih.gov/books/NBK115122/

74 Gentileschi S, Servillo M, Garganese G, et al. The lymphatic superficial circumflex iliac vessels deep branch perforator flap: A new preventive approach to lower limb lymphedema after groin dissection-preliminary evidence. Microsurgery 2017;37(06): 564-573

75 Rosenthal R, Kasenda B, Dell-Kuster S, et al. Completion and publication rates of randomized controlled trials in surgery: An empirical study. Ann Surg 2015;262(01):68-73 